\title{
MATHEMATICAL MODELING OF TUMOR CELL GROWTH AND IMMUNE SYSTEM INTERACTIONS
}

\author{
FATHALLA A. RIHAN* \\ Department of Mathematical Sciences, United Arab Emirates University, Al-Ain, 1755, UAE \\ frihan@uaeu.ac.ae \\ MUNTASER SAFAN \\ Department of Mathematics, Faculty of Science, Mansoura University, Mansoura, Egypt \\ MOHAMED A. ABDEEN \\ Department of Mathematics, Helwan University, Cairo, Egypt \\ DUAA H. ABDEL-RAHMAN \\ Department of Mathematical Sciences, United Arab Emirates University, Al-Ain, 1755, UAE
}

\begin{abstract}
In this paper, we provide a family of ordinary and delay differential equations to describe the dynamics of tumor-growth and immunotherapy interactions. We explore the effects of adoptive cellular immunotherapy on the model and describe under what circumstances the tumor can be eliminated. The possibility of clearing the tumor, with a strategy, is based on two parameters in the model: the rate of influx of the effector cells, and the rate of influx of IL2. The critical tumor-growth rate, below which endemic tumor does not exist, has been found. One can use the model to make predictions about tumor-dormancy.
\end{abstract}

Keywords: Bifurcation; mathematical modeling; immunotherapy; Interleukin-2; steady states; time-lags.

\section{Introduction}

Cancer is one of the most difficult diseases to be treated clinically, and one of the main causes of death. Accordingly, a great research effort is being devoted to understand the interaction between the tumor cells and the immune system. Mathematical models, using ordinary, partial, and delay differential equations, ${ }^{19}$ play an important role in understanding the dynamics and tracking tumor and immune populations over time. Although the theoretical study of tumor immune dynamics

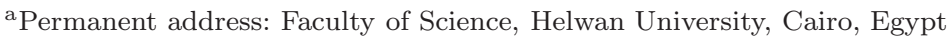


has a long history, ${ }^{1,7,8}$ the multifaceted nature of cancer requires sophisticated, nonlinear mathematical models to capture more realistic growth dynamics.

The treatment of cancer is then one of the most challenging problems of modern medicine. The treatment should satisfy two basic conditions: First, it should destroy cancer cells in the entire body. Second, it should distinguish between cancerous and healthy cells. Other treatments such as surgery and/or chemo-and radiotherapies have played key roles in treatment, but in many cases they do not represent a cure. Immunotherapy seems to be the method that best fulfils both of these requirements. ${ }^{3,5,6,14-16}$

Numerous research papers have been made to explore the effects of the immune system in eliminating the tumor cells in the host, by stimulating the host's own immune response to kill cancer cells. ${ }^{17}$ When tumor cells appear in a body, the immune system tries to identify and then eliminate them. Immunotherapy refers to the use of cytokines usually together with Adoptive Cellular Immunotherapy (ACI). Cytokines are protein hormones that mediate both natural and specific immunity. They are produced mainly by activated $\mathrm{T}$ cells (lymphocytes) during cellular-mediated immunity. Interleukin-2 (IL-2) is the main cytokine responsible for lymphocyte activation, growth and differentiation. IL-2 has been shown to enhance Cytotoxic T Cells (CTL) activity at different disease stages. However, ACI refers to the injection of cultured immune cells that have anti-tumor reactivity into tumor bearing host. This interaction is analyzed and studied in various levels of bio-mathematical researches. They commonly focused on the models on ODEs over time. For example, in 1985, De Boer et al. ${ }^{7}$ suggested a rather complex model in the experiments which contains eleven ordinary differential equations with five algebraic equations to describe anti-tumor response with the help of IL-2. A simple version of this model is proposed by Kirschner and Panetta. ${ }^{15}$ The model is only based on three differential equations. Further analysis by several authors has also been done; See Refs. 2, 5, 8, 11,13 .

In this paper, we investigate the dynamics and bifurcation analysis of a mathematical model for the tumor growth - immunotherapy interactions (in vitro). In Section 2, we provide different bilinear models, using ODEs and DDEs, to describe the response of the effector cells to the growth of tumor cells. In Section 3, we study the local stability of the steady states for tumor-free and endemic persistence. Bifurcation analysis for a three-equations model and finding regions of existence of the equilibria are discussed in Section 4. In Section 5, we discuss the conditions that ensure tumor-clearance possibilities, and conclude in Section 6.

\section{Bilinear Models}

The model of Kuznetsov and Taylors ${ }^{16}$ describes the response of the Effector Cells (ECs) to the growth of Tumor Cells (TCs). The assumption in this model is that $E$, $T, C, E^{*}$ and $T^{*}$ denote the local concentrations of ECs, TCs, EC-TC complexes, inactivated TCs and lethally hit TCs, respectively. The rate of binding of ECs 
to TCs and the rate of separation of ECs from TCs without damaging them are denoted by $k_{1}$ and $k_{-1}$, respectively. The rate at which EC-TC integrations program for lysis is denoted by $k_{2}$ while the rate at which EC-TC interaction inactivate ECs is denoted by $k_{3}$. The model takes the form:

$$
\begin{aligned}
\frac{d E}{d t} & =s+F(T, C)-d_{1} E-k_{1} E T+\left(k_{-1}+k_{2}\right) C, \\
\frac{d T}{d t} & =a T(1-b T)-k_{1} E T+\left(k_{-1}+k_{3}\right) C, \\
\frac{d C}{d t} & =k_{1} E T-\left(k_{-1}+k_{2}+k_{3}\right) C, \\
\frac{d E^{*}}{d t} & =a k_{3} C-d_{3} E^{*}, \\
\frac{d T^{*}}{d t} & =k_{2} C-d_{3} T^{*} .
\end{aligned}
$$

Here, $s$ represents the normal rate (not increased by the presence of the tumor) of the flow of adult ECs into the tumor site, $F(C, T)$ describes the accumulation of ECs in the tumor site. While, $d_{1}, d_{2}$ and $d_{3}$ are the coefficients of the processes of destruction and migration of $E, E^{*}$ and $T^{*}$, respectively. The maximal growth of tumor is represented by the coefficient $a$, and $b$ is the environment capacity. It was suggested in ${ }^{16}$ that the function $F$ takes the form

$$
F(C, T)=F(E, T)=\frac{p E T}{r+T},
$$

where $p$ and $r$ are positive constants.

The idea in this paper is to simplify the above model and reduce it into a two- or three-equations model to describe the interactions of three type of cell populations: the activated immune-system cells, E(t) (or effector cells such as cytotoxic T-cells, macrophages, and natural killer cells that are cytotoxic to the tumor cells); the tumor cells, T(t); and the concentration of IL-2 in the single tumor-site compartment, $I_{L}(t)$. The above model can then governed by the three equations (see Ref. 15):

$$
\begin{aligned}
& \frac{d E}{d t}=\bar{c} T-\overline{\mu_{1}} E+\overline{\theta_{1}} E I_{L}+\overline{s_{1}}, \\
& \frac{d T}{d t}=\overline{r_{2}} T(1-\bar{b} T)-\bar{\alpha} E T, \\
& \frac{d I_{L}}{d t}=\overline{\theta_{2}} E T-\overline{\mu_{2}} I_{L}+\overline{s_{2}},
\end{aligned}
$$

with initial conditions: $E(0)=E_{0}, \quad T(0)=T_{0}, \quad I_{L}(0)=I_{L_{0}}$, where $\bar{c}$ is the antigenicity rate of the tumor, $\overline{s_{1}}$ is the external source of the effector cells, with 
rate of death $\overline{\mu_{1}}$. Whereas the parameter $\overline{r_{2}}$ incorporates both multiplication and death of tumor cells. The maximal carrying capacity of the biological environment for tumor cell is $\bar{b}^{-1}$; and $\overline{\theta_{1}}$ is considered as the cooperation rate of effector cells with Interleukin-2 parameter, $\bar{\alpha}$ is the rate of tumor cells, and $\overline{\theta_{2}}$ is the competition rate between the effector cells and the tumor cells. External input of IL-2 into the system is $\overline{s_{2}}$ and the rate loss parameter of effector cells is $\overline{\mu_{2}}$.

\subsection{Non-dimensionalization}

System (2) is a stiff model, and needs a special care in the analysis and numerical treatment. The state variables are very sensitive to small perturbations (or changes) in the parameters occur in the model. In the meantime, the parameter estimates are also sensitive to the noisy data and observations. To ease the analysis and stability of the steady states with meaningful parameters and less sensitive (or rubus) model, we non-dimensionalize the bilinear model (2), by taking the following scaling:

$$
\begin{gathered}
x=\frac{E}{E_{0}}, y=\frac{T}{T_{0}}, z=\frac{I_{L}}{I_{L_{0}}}, \tau=t_{s} t, c=\frac{\bar{c} T_{0}}{t_{s} E_{0}}, \theta_{1}=\frac{\overline{\theta_{1}} I_{L_{0}}}{t_{s}}, \mu_{1}=\frac{\overline{\mu_{1}}}{t_{s}}, \\
b=\bar{b} T_{0}, r_{2}=\frac{\overline{r_{2}}}{t_{s}}, \alpha=\frac{\bar{\alpha} E_{0}}{t_{s}}, \mu_{2}=\frac{\overline{\mu_{2}}}{t_{s}}, \theta_{2}=\frac{\overline{\theta_{2}} E_{0} T_{0}}{t_{s} I_{L_{0}}}, s_{1}=\frac{\overline{s_{1}}}{t_{s} E_{0}}, s_{2}=\frac{\overline{s_{2}}}{t_{s} I_{L_{0}}} .
\end{gathered}
$$

Therefore, after the above substitution into (2) and replacing $\tau$ by $t$, the model becomes

$$
\begin{aligned}
& \frac{d x}{d t}=c y-\mu_{1} x+\theta_{1} x z+s_{1}, \\
& \frac{d y}{d t}=r_{2} y(1-b y)-\alpha x y \\
& \frac{d z}{d t}=\theta_{1} x y-\mu_{2} z+s_{2}
\end{aligned}
$$

with initial conditions: $x(0)=x_{0}, y(0)=y_{0}, z(0)=z_{0}$. Here $x(t), y(t)$ and $z(t)$ denote the dimensionless density of ECs, TCs and LI-2, respectively. In model (4), there are four possible cases of treatments, according the values of $s_{1}$ and $s_{2}$ : (i) no-treatment case $\left(s_{1}=s_{2}=0\right)$; (ii) adoptive cellular immunotherapy case $\left(s_{1}>0, s_{2}=0\right)$; (iii) Interleukin-2 case $\left(s_{1}=0, s_{2}>0\right)$; (iv) and Immunotherapy with both Adoptive Cellular Immunotherapy (ACI) and IL-2 $\left(s_{1}>0, s_{2}>0\right)$.

Yafia $^{22}$ simplified system (4) into a Lotka-Volterra form

$$
\begin{aligned}
& \frac{d x}{d t}=\omega x y-\mu x+s, \\
& \frac{d y}{d t}=r y(1-b y)-x y,
\end{aligned}
$$

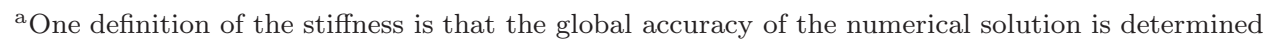
by stability rather than local error and implicit methods are more appropriate for it. 
where $\omega$ is immune response to the appearance of the TCs, and $s$ has the same meaning of $s_{1}, r$ has the meaning of $r_{2}$ and $\mu$ has the meaning of $\mu_{1}$ in the above model. If we consider a time delay $\tau>0$ in (5) due to the time-lag in the interaction between ECs and TCs, the model takes the form

$$
\begin{aligned}
& \frac{d x}{d t}=\omega x(t) y(t-\tau)-\mu x(t)+s \\
& \frac{d y}{d t}=r y(t-\tau)(1-b y(t))-x(t) y(t) .
\end{aligned}
$$

To solve model (6), we should provide an initial function with initial function $y(t)=$ $\psi(t), \quad t \in[-\tau, 0]$ instead of the initial value $y(0)$ at $t=0$. It has been shown that model (6) has visible and bounded solution $\left(\right.$ see $\left.^{11,23}\right)$. When the time delay is included in the simplified model (5), the state of returning tumor cells can be observed, as DDE models have reacher dynamics than do ODE models; see the graphs displayed in Figures 1, 2 and 3. We next study the stability of the steady states of the above models, according the values of the parameters given in Table 1 .
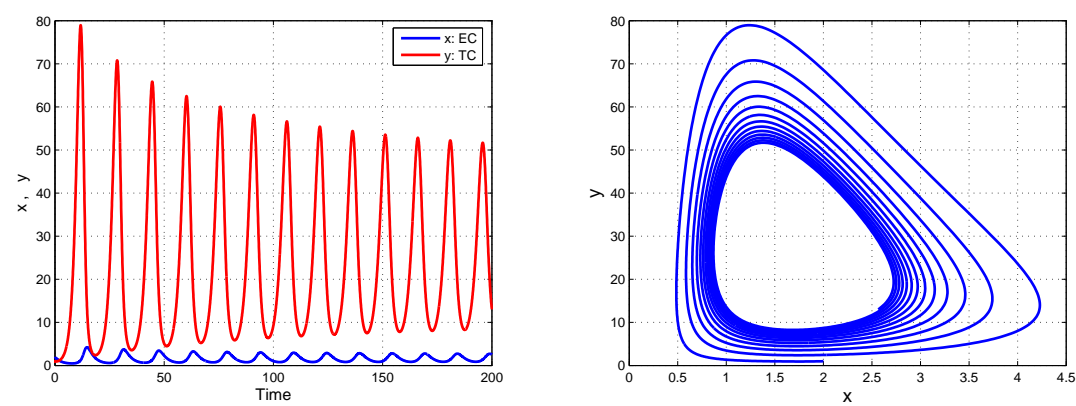

Fig. 1. Solution of the DDEs (6) when $\omega=0.01184, \mu=0.3747, s=0.1181, r=1.636, b=0.002$ and $\tau=0.8$. This shows a unstable endemic equilibrium.
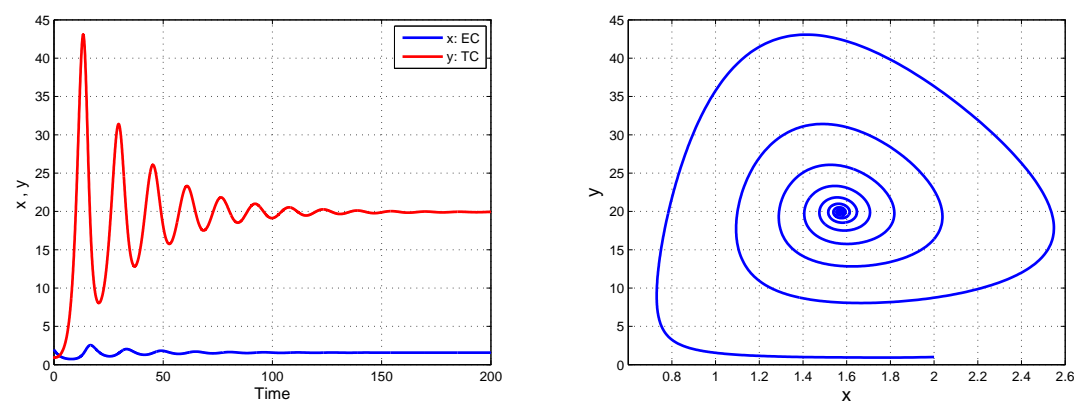

Fig. 2. Solution of the DDEs (6) when $\omega=0.01184, \mu=0.3747, s=0.2181, r=1.636, b=0.002$ and $\tau=0.8$. This shows a stable endemic equilibrium. 

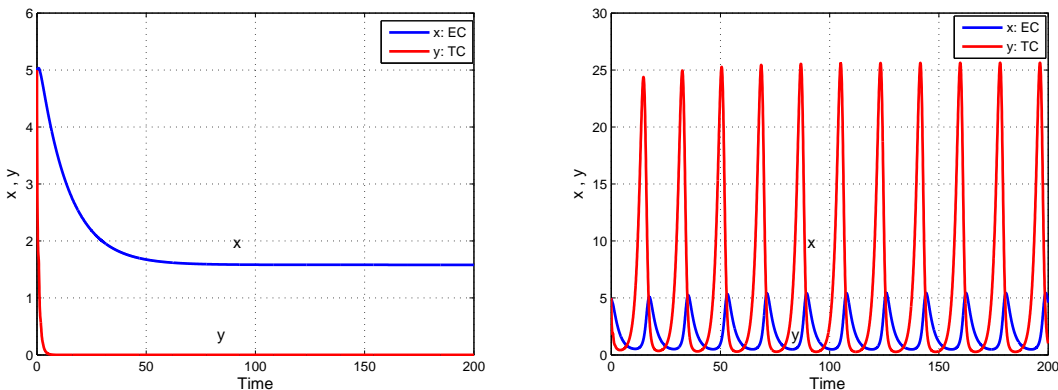

Fig. 3. Solution of the DDEs (6) when $\omega=0.04184, \mu=0.03747$ (left), and $\mu=0.3747$ (right), $s=0.2181, r=1.636, b=0.002$ and $\tau=0.8$. The infection-free equilibrium is asymptotically stable in the left banner and unstable in the right banner.

Table 1. The non-dimensionalization parameters of bilinear model (4).

\begin{tabular}{|ccc|}
\hline$c=5 / 18$ & $\mu_{1}=1 / 6$ & $\theta_{1}=1 / 18000000$ \\
$r_{2}=1$ & $b=1 / 1000$ & \\
$\mu_{2}=500 / 9$ & $\theta_{2}=250 / 9$ & \\
\hline
\end{tabular}

\section{Steady States and Stability}

The solutions of practical interest should have non-negative population $x, y, z$. However, it is hard to find a closed analytical solution for the above nonlinear models, instated we can study their qualitative behavior by studying the stability of the steady states. We then assume that the parameters occur in the models are also non-negative.

\subsection{Tumor-free equilibrium and its stability}

To ease the analysis we start with the 2-population model (5). The steady states of the reduced model (5) are the intersection of the null-clines $\frac{d x}{d t}=0$ and $\frac{d y}{d t}=0$. If $y=0$, the free tumor equilibrium is at $(\overline{\mathrm{x}}, \overline{\mathrm{y}})=\left(\frac{s}{\mu}, 0\right)$ This steady state is always exist, since $\frac{s}{\mu}>0$. It is clear that the tumor-free equilibrium $E_{0}=\left(x^{*}, y^{*}\right)=\left(\frac{s}{\mu}, 0\right)$ of the model (5) is asymptotically stable if $r \mu<s$, and unstable if $r \mu>s$. Whoever, when we consider the DDEs model, the characteristic equation of the linearized model of (6) at $E_{0}=\left(\frac{s}{\mu}, 0\right)$ takes the form

$$
(\lambda+r)\left(\lambda-e^{-\lambda \tau}+\frac{s}{\mu}\right)=0 .
$$


When $\tau=0$, it is clear that $E_{0}$ is asymptotically stable when $r \mu<s$ and unstable otherwise. However, if $\tau>0$, equation (7) has a negative real root $\lambda=-r$ and roots of

$$
\lambda-e^{-\lambda \tau}+\frac{s}{\mu}=0 .
$$

Put $\lambda=\xi i$ in (8) and separate real and imaginary parts yields

$$
\xi^{2}=\left[r^{2}-\left(\frac{s}{\mu}\right)^{2}\right]
$$

Therefore, when $|r \mu|<|s|$ there are no positive real root $\xi$. This shows that all the roots of $(7)$ have negative real parts and $E_{0}$ is asymptotically stable.

In case of the three-equations model (4), and at the equilibrium points, we have

$$
0=c y-\mu_{1} x+\theta_{1} x z+s_{1}, \quad 0=r_{2} y(1-b y)-\alpha x y, \quad 0=\theta_{2} x y-\mu_{2} z+s_{2} .
$$

Putting $y=0$, yields the tumor-free equilibrium, namely

$$
E_{0}=\left[s_{1} \mu_{2} /\left(\mu_{1} \mu_{2}-\theta_{1} s_{2}\right), 0, s_{2} / \mu_{2}\right] .
$$

It is clear that the infection-free equilibrium $E_{0}$ exists if and only if $s_{2}<\mu_{1} \mu_{2} / \theta_{1}$. Therefore, we restrict our analysis to the case where $s_{2}<\mu_{1} \mu_{2} / \theta_{1}$. To study its stability, we consider the corresponding Jacobian matrix

$$
J_{E_{0}}=\left[\begin{array}{ccc}
-\mu_{1}+\theta_{1} s_{2} / \mu_{2} & c & \theta_{1} s_{1} \mu_{2} /\left(\mu_{1} \mu_{2}-\theta_{1} s_{2}\right) \\
0 & r_{2}-\alpha s_{1} \mu_{2} /\left(\mu_{1} \mu_{2}-\theta_{1} s_{2}\right) & 0 \\
0 & \theta_{2} s_{1} \mu_{2} /\left(\mu_{1} \mu_{2}-\theta_{1} s_{2}\right) & -\mu_{2}
\end{array}\right] .
$$

It has the eigenvalues $-\mu_{1}+\theta_{1} s_{2} / \mu_{2}, r_{2}-\alpha s_{1} \mu_{2} /\left(\mu_{1} \mu_{2}-\theta_{1} s_{2}\right)$ and $-\mu_{2}$. Therefore $E_{0}$ is locally asymptotically stable if and only if $r_{2}<\alpha s_{1} \mu_{2} /\left(\mu_{1} \mu_{2}-\theta_{1} s_{2}\right)$, while otherwise it is unstable saddle-point.

\subsection{Endemic equilibrium and its stability}

Consider again the two-equations model (5). If $y \neq 0$, the steady states are obtained by solving $\omega r b y^{2}-r(\omega+\mu b) y+\mu r-s=0$. In this case we have two endemic equilibria $P_{1}=\left(x_{1}, y_{1}\right)$ and $P_{2}=\left(x_{2}, y_{2}\right)$, where

$$
\begin{array}{ll}
x_{1}=\frac{-r(b \mu-\omega)-\sqrt{\triangle}}{2 \omega}, & y_{1}=\frac{r(b \mu+\omega)+\sqrt{\triangle}}{2 r b \omega} \\
x_{2}=\frac{-r(b \mu-\omega)+\sqrt{\triangle}}{2 \omega}, & y_{2}=\frac{r(b \mu+\omega)-\sqrt{\triangle}}{2 r b \omega}
\end{array}
$$

with $\triangle=r^{2}(b \mu-\omega)^{2}+4 \omega r b \mu>0$. The Jacobian matrix of the system (5) at the endemic equilibrium $P_{1}$ is

$$
J_{\text {endemic }}=\left[\begin{array}{cc}
\omega y_{1}-\mu & \omega x_{1} \\
-y_{1} & r-2 b r y_{1}-x_{1}
\end{array}\right] .
$$

Prop 3.1. If the endemic equilibrium $P_{1}$ exists and has nonnegative coordinates, then $\operatorname{tr}\left(J_{\text {endemic }}\right)>0$ and $P_{1}$ is unstable. 


\section{Proof: Since}

$$
\operatorname{tr}\left(J_{\text {endemic }}\right)=\frac{\omega^{2}-\omega(r b+b \mu)-r b^{2} \mu}{2 b \omega}+\frac{\omega-r b}{2 r b \omega} \sqrt{r^{2}(b \mu+\omega)^{2}-4 r b \omega(r \mu-s)},
$$

then inequality $\operatorname{tr}\left(J_{\text {endemic }}\right)>0$ is true if

$$
r\left[\omega^{2}-\omega(r b+b \mu)-r b^{2} \mu\right]>(r b-\omega) \sqrt{r^{2}(b \mu+\omega)^{2}-4 r b \omega(r \mu-s)} .
$$

Therefore, when $r \mu<s$ and $\omega<-b \mu$, we have $\omega^{2}-\omega b(r+\mu)-r \mu b^{2}>0$ and hence both sides of the inequality are positive. Therefore if the point $P_{1}$ exists and has nonnegative coordinates, then $\operatorname{tr}\left(J_{\text {endemic }}\right)>0$ and the point $P_{1}$ is unstable whenever $\omega<-b \mu$ and $r \mu<s$.

Similarly, it is easy to prove the following proportion.

Prop 3.2. If the point $P_{2}$ exists and has nonnegative coordinates, then it is asymptotically stable.

We extend the above analysis to the case of the three-equations model (4). The tumor-persistent solutions are obtained by putting $y \neq 0$ and omitting $x$ and $z$ in (9) to get a scalar equation in the variable $y$ which reads

$$
F\left(r_{2}, y\right)=C_{3} y^{3}+C_{2} y^{2}+C_{1} y+C_{0}=0
$$

where

$$
\begin{aligned}
& C_{3}=\theta_{1} \theta_{2} r_{2}^{2} b^{2}>0, \\
& C_{2}=-2 \theta_{1} \theta_{2} b r_{2}^{2}<0, \\
& C_{1}=r_{2}^{2} \theta_{1} \theta_{2}+r_{2} b\left(\mu_{1} \mu_{2}-s_{2} \theta_{1}\right) \alpha+c \mu_{2} \alpha^{2}, \\
& C_{0}=\alpha\left[r_{2}\left(\theta_{1} s_{2}-\mu_{1} \mu_{2}\right)+\mu_{2} s_{1} \alpha\right] .
\end{aligned}
$$

Since $s_{2}<\mu_{1} \mu_{2} / \theta_{1}$, then the coefficient $C_{1}$ is always positive, while the coefficient $C_{0}$ can take positive and negative values depending on the values of the model parameters. Also, equation (12) is well-defined for all $y \in[0,1 / b]$. Its left hand side is a polynomial of degree three, and its zeros are not easy to be obtained in a closed-form. However, some conditions in the parameters occur in the model to ensure the existence of its solutions could be deduced. The Equation (12) can also be seen as a bifurcation equation in $r_{2}$ and $y$ where we keep all other parameters fixed. Once a solution $y>0$ of this equation has been obtained, we could find positive $x$ and $z$ from the other equations in (9). Therefore, there is a one-to-one correspondence between the solutions of (12) and the endemic stationary solutions. for more insights, we next study the bifurcation analysis.

\section{Bifurcation Analysis of Model (4)}

The bifurcation analysis gives a deeper analysis about the model. It answers the query that "how does the behavior of the solutions change as parameters change". We restrict ourselves to only study the bifurcation analysis of ODEs models rather than DDEs models. 


\subsection{Bifurcation points for the parameter $r_{2}$}

In this subsection, we then analyze the bifurcation so that the tumor growth rate $r_{2}$ acts as a bifurcation parameter. Therefore, to find the bifurcation point(s) we put $y=0$ in equation (12) to get $r_{2}=\alpha \mu_{2} s_{1} /\left(\mu_{1} \mu_{2}-\theta_{1} s_{2}\right):=\bar{r}_{2}$. Hence, there is only one transcritical bifurcation point at

$$
\left(\bar{r}_{2}, \bar{y}\right)=\left(\frac{\alpha \mu_{2} s_{1}}{\mu_{1} \mu_{2}-\theta_{1} s_{2}}, 0\right) .
$$

Now, we compute the direction of bifurcation at $\left(\bar{r}_{2}, 0\right)$ so that

$$
\left.\frac{d y}{d r_{2}}\right|_{\left(\bar{r}_{2}, 0\right)}=-\left.\frac{F_{r_{2}}}{F_{y}}\right|_{\left(\bar{r}_{2}, 0\right)}
$$

where

$$
\left.F_{r_{2}}\right|_{\left(\bar{r}_{2}, 0\right)}=-\alpha\left(\mu_{1} \mu_{2}-\theta_{1} s_{2}\right)<0,\left.F_{y}\right|_{\left(\bar{r}_{2}, 0\right)}=\left(c+b s_{1}+\frac{\theta_{1} \theta_{2} \mu_{2} s_{1}^{2}}{\left(\mu_{1} \mu_{2}-\theta_{1} s_{2}\right)^{2}}\right) \mu_{2} \alpha^{2}>0 .
$$

Hence, the bifurcation at the point $\left(\bar{r}_{2}, 0\right)$ is forward, irrespective of the values of the model-parameters. We notice that the model we consider here has only one bifurcation point $\left(\bar{r}_{2}, 0\right)$ at which the bifurcation is forward.

\subsection{Bifurcation diagrams for the parameter $\alpha$}

The parameter $\alpha$ is very important in the model that plays an effective role to define cancer behavior. We investigate numerically, in this subsection, the bifurcation of the model for the parameter $\alpha$. We consider the four cases: No-treatment case $\left(s_{1}=\right.$ $\left.s_{2}=0\right)$, Adoptive Cellular Immunotherapy case $\left(s_{1}>0, s_{2}=0\right)$, Interleukin-2 case $\left(s_{1}=0, s_{2}>0\right)$ and immunotherapy with both ACI and IL-2 case $\left(s_{1}>0, s_{2}>0\right)$.

If we solve $F(y, \alpha)=F(\alpha, y)=0$ in $\alpha$, we have

$$
\begin{aligned}
& \alpha_{+}=\frac{r_{2} b\left(y-\frac{1}{b}\right)}{2 \mu_{2}\left(c y+s_{1}\right)}\left[\left(\theta_{1} s_{2}-\mu_{1} \mu_{2}\right)+\sqrt{\left(\mu_{1} \mu_{2}-s_{2} \theta_{1}\right)^{2}-4 \theta_{1} \theta_{2} \mu_{2} y\left(c y+s_{1}\right)}\right], \\
& \alpha_{-}=\frac{r_{2} b\left(y-\frac{1}{b}\right)}{2 \mu_{2}\left(c y+s_{1}\right)}\left[\left(\theta_{1} s_{2}-\mu_{1} \mu_{2}\right)-\sqrt{\left(\mu_{1} \mu_{2}-s_{2} \theta_{1}\right)^{2}-4 \theta_{1} \theta_{2} \mu_{2} y\left(c y+s_{1}\right)}\right] .
\end{aligned}
$$

To plot $(\alpha, y)$ in the interval $0<y<1 / b$, under the conditions that

$$
\begin{aligned}
& \left(\mu_{1} \mu_{2}-s_{2} \theta_{1}\right)^{2}-4 \theta_{1} \theta_{2} \mu_{2} y\left(c y+s_{1}\right)>0, \\
& -4 \theta_{1} \theta_{2} c \mu_{2} y^{2}-4 \theta_{1} \theta_{2} \mu_{2} s_{1} y+\left(\mu_{1} \mu_{2}-s_{2} \theta_{1}\right)^{2}>0,
\end{aligned}
$$

we have

$$
y^{2}+\frac{s_{1}}{c} y-\frac{\left(\mu_{1} \mu_{2}-s_{2} \theta_{1}\right)^{2}}{4 \theta_{1} \theta_{2} c \mu_{2}}<0
$$

Then

$\frac{1}{2}\left[\frac{-s_{1}}{c}-\sqrt{\left(\frac{s_{1}}{c}\right)^{2}+\frac{\left(\mu_{1} \mu_{2}-s_{2} \theta_{1}\right)^{2}}{\theta_{1} \theta_{2} c \mu_{2}}}\right]<y<\frac{1}{2}\left[\frac{-s_{1}}{c}+\sqrt{\left(\frac{s_{1}}{c}\right)^{2}+\frac{\left(\mu_{1} \mu_{2}-s_{2} \theta_{1}\right)^{2}}{\theta_{1} \theta_{2} c \mu_{2}}}\right]$. 
For $0<y<y_{+}$, where

$$
y_{+}=\frac{1}{2}\left[\frac{-s_{1}}{c}+\sqrt{\left(\frac{s_{1}}{c}\right)^{2}+\frac{\left(\mu_{1} \mu_{2}-s_{2} \theta_{1}\right)^{2}}{\theta_{1} \theta_{2} c \mu_{2}}}\right],
$$

we have two cases $y_{+}<1 / b$ or $y_{+}>1 / b$. The graphs in Figure 4 , which are obtained numerically, display the bifurcation diagrams for different cases, where (i) $s_{1}=s_{2}=0$, (ii) $s_{1}=10, s_{2}=0$, (iii) $s_{1}=0, s_{2}=40$ and (vi) $s_{1}=10, s_{2}=40$.

Given the threshold point $(\alpha, y)=\left(\alpha^{*}, 0\right)$, the tumor clearance condition is $\alpha>\alpha^{*}$, where

$$
\alpha^{*}=\frac{\left(\mu_{1} \mu_{2}-\theta_{1} s_{2}\right) r_{2}}{\mu_{2} s_{1}} .
$$

Therefore, when $s_{1}>0$ then $\alpha^{*}>0$ and $\alpha \propto 1 / s_{1}$. Thus, we can arrive to tumor clearance quickly when the value of $s_{1}$ increases. We notice from Figure 4 that the locations of saddle node bifurcation points $\mathbf{A}$ and $\mathbf{B}$ bridge the one-positive equilibrium to the three-positive equilibria. The supercritical hopf bifurcation point $\mathbf{C}$ joints between existence of stable limit cycles and non-existence of limit cycles. The transcritical bifurcation point $\mathbf{D}$ at $(\alpha, y)=\left(\alpha^{*}, 0\right)$ bridges the one-positive equilibrium and no-positive equilibria.

\subsection{Regions of existence of the equilibria}

In addition to the tumor-free equilibrium, equation (12) may have one to three persistent-tumor equilibria, depending on the values of the model-parameters. However, before we proceed we provide the following proposition which is helpful in the analysis.

Prop 4.1. Equation (12) does not have two persistent-tumor equilibria if $r_{2}<\bar{r}_{2}$, where $\bar{r}_{2}$ is given in (13).

Proof: Since the bifurcation direction at the point $\left(\bar{r}_{2}, 0\right)$ is always forward, then equation (12) has two positive roots, for $r_{2}<\bar{r}_{2}$, if and only if $F\left(\bar{r}_{2}, y\right)=$ $y\left(\bar{C}_{3} y^{2}+\bar{C}_{2} y+\bar{C}_{1}\right)=0$ has two positive zeros, where

$$
\begin{aligned}
& \bar{C}_{2}=\frac{-2}{b} \bar{C}_{3}=-2 b \theta_{1} \theta_{2}\left(\frac{\mu_{2} s_{1} \alpha}{\mu_{1} \mu_{2}-\theta_{1} s_{2}}\right)^{2}, \\
& \bar{C}_{1}=\theta_{1} \theta_{2}\left(\frac{\mu_{2} s_{1} \alpha}{\mu_{1} \mu_{2}-\theta_{1} s_{2}}\right)^{2}+\mu_{2} \alpha^{2}\left(b s_{1}+c \mu_{2}\right) .
\end{aligned}
$$

However, $\bar{C}_{2}^{2}-4 \bar{C}_{1} \bar{C}_{3}=-4 \bar{C}_{3} \mu_{2} \alpha^{2}\left(b s_{1}+c \mu_{2}\right)<0$. Therefore, the proof is complete.

Now, to find the conditions, on the model-parameters, being required for the existence of the persistent equilibria we make the use of both Descant's rule of signs and the Sturm sequence. ${ }^{4}$ In equation (12), it is clear that the coefficients $C_{3}, C_{2}$ and $C_{1}$ have fixed signs, while the coefficient $C_{0}$ can take positive or negative 


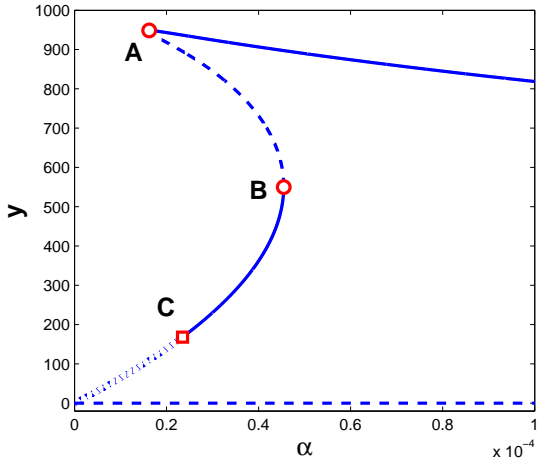

(a) No-Treatment Case, $s_{1}=s_{2}=0$

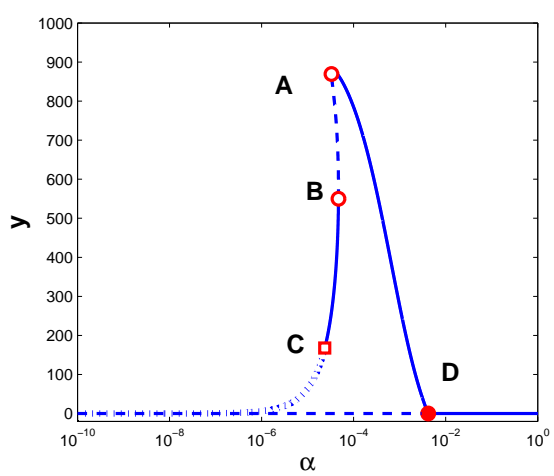

(c) Adoptive cellular immunotherapy case, $s_{1}=40, s_{2}=0$.

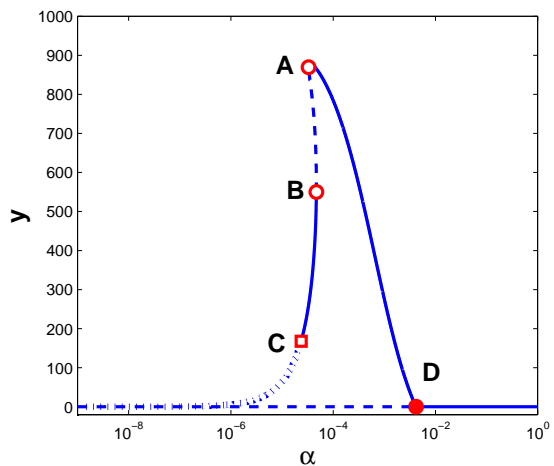

(e) Immunotherapy with both ACI and IL-2 $s_{1}=40, s_{2}=10$.

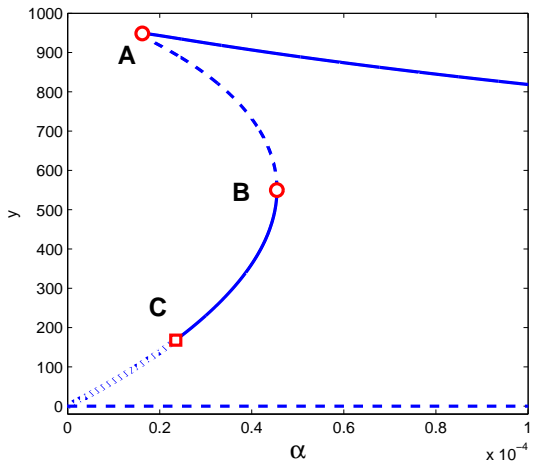

(b) Interleukin-2 Case $s_{1}=0, s_{2}=10$

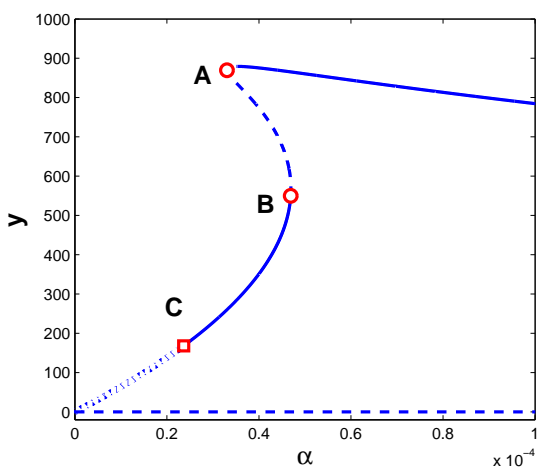

(d) Small interval of $\alpha$ for ACI Case, $s_{1}=$ $40, s_{2}=0$.

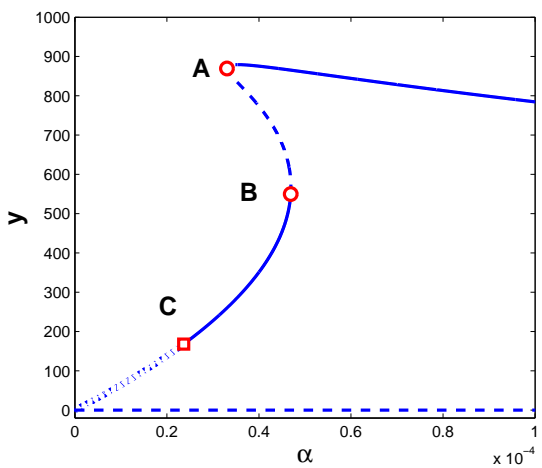

(f) Small interval of $\alpha$ for immunotherapy with both ACI and IL-2 case, $s_{1}=40, s_{2}=10$.

Fig. 4. Shows the bifurcation diagrams for the bilinear model (4) for the parameter $\alpha$ : [-] represents the stable equilibrium, [- - ] represents the unstable equilibrium, [...] is the stable

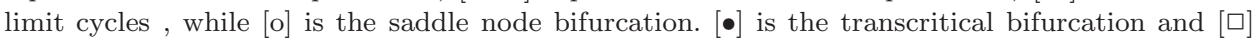
supercritical hopf bifurcation. The values of parameters are given in Table 1. 
values. Hence, the number of feasible tumor-persistent equilibria (on the interval $y \in[0,1 / b])$ depends on the difference between the number of sign changes at $y=0$ and at $y=1 / b$ in the Sturm sequence $\left\{P_{0}(y), P_{1}(y), P_{2}(y), P_{3}(y)\right\}$, such that

$$
\begin{aligned}
P_{0}(y) & =F(y)=C_{3} y^{3}+C_{2} y^{2}+C_{1} y+C_{0}, \\
P_{1}(y) & =F^{\prime}(y)=3 C_{3} y^{2}+2 C_{2} y+C_{1}, \\
P_{2}(y) & =\frac{2}{9} R y+\frac{1}{9} S, \quad P_{3}(y)=T, \quad \text { where } R=\left(C_{2}^{2}-3 C_{1} C_{3}\right) / C_{3}, \\
S & =\left(C_{2} C_{1}-9 C_{0} C_{3}\right) / C_{3}, T=\frac{R}{3}-3 C_{3}\left(\frac{S}{2 R}-\frac{C_{2}}{3 C_{3}}\right)^{2} .
\end{aligned}
$$

Hence the number of sign changes depends on the sign of the coefficient $C_{0}$ and the remainders $R, S$, and $T$. Table 2 shows the conditions required for the existence of persistent-tumor equilibria as well as their numbers, where we take into account that $C_{0}>0$ implies $S<0$.

Table 2. The number of positive steady states (SS) are determined by the signs of the coefficients (12) and the signs of the quantities $R, S$ and $T$ from the Sturm sequence. Blank entries correspond to coefficients which may take positive, negative or zero values.

\begin{tabular}{|c||c|ccc|}
\hline SS & $C_{0}$ & $R$ & $S$ & $T$ \\
\hline 0 & + & & - & - \\
\hline 1 & - & & & - \\
\hline 3 & - & + & - & + \\
\hline
\end{tabular}

We may note that the tumor-persistent equilibria do not exist for $C_{0}>0$, while one or three equilibria exist depending on the other relevant quantities. The interest is to find the area where the tumor-free equilibrium is a global attractor. Based on proposition 4.1 and Table 2 , this area is determined by $C_{0}>0$ that is equivalent to

$$
r_{2}<\frac{\mu_{2} s_{1} \alpha}{\mu_{1} \mu_{2}-\theta_{1} s_{2}}:=r_{2}^{\star},
$$

where $r_{2}^{\star}$ is the critical growth rate of the tumor cell population, separating between nonexistence and existence of positive endemic equilibria.

If we consider the general case of immunotherapy with both ACI and IL-2 treatments, then according to the conditions given in Table 2 and data displayed in Figure 4, then Figure 5 displays six stability regions in terms of the two parameters $\alpha, r_{2}$ (according to the number of positive equilibria and the limit cycles). However, Figure 6 shows phase spaces for different equilibria where there are stable steady states, unstable steady states, stable manifold, unstable manifold and initial conditions. For example, Figure 6.f shows the trajectories where there are three tumor-persistent equilibria for which two of them are locally stable and one, lying 


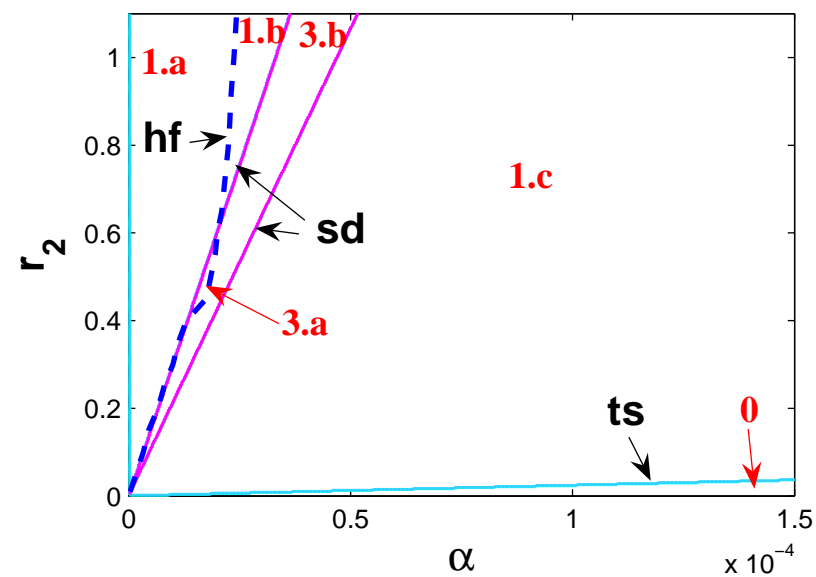

Fig. 5. The two-dimensional transition structure as a function of $\alpha$ (the tumor cell is predated by effector cells rate), and $r_{2}$ (the maximal growth rate of the tumor cells population): sd is the saddle node bifurcation, ts is the transcritical bifurcation and hf is the supercritical hopf bifurcation. (Immunotherapy with both ACI and IL-2 Case; See Table 1 and $s_{1}=40, s_{2}=10$.)

in between, for tumor-free equilibrium is unstable (that correspond to region $\mathbf{3 . b}$ in Figure 5). Figure 6.e shows the trajectories where there are three tumor-persistent equilibria for which one of them is locally stable, one is stable limit cycles and one, lying in between, for tumor-free equilibrium is unstable (that correspond to region 3.a in Figure 5).

\section{Tumor-Clearance Possibilities}

Let us introduce the following definition to facilitate the analysis.

Definition 5.1. The threshold parameter $\mathcal{R}_{0}$ (the minimum tumor-clearance parameter) is the parameter that has the property that if $\mathcal{R}_{0}<1$, then the endemic tumor does not exist, while if $\mathcal{R}_{0}>1$ the tumor persists $\left(\mathrm{see}^{21}\right)$.

The parameter $\mathcal{R}_{0}$ can then be expressed in terms of the ratio between the tumorgrowth rate and the critical tumor-growth rate separating between non-existence and existence of endemic tumor. Now, for the three-equations model, the tumorfree equilibrium is the unique equilibrium if and only if $r_{2}<r_{2}^{\star}$, where it is also locally asymptotically stable. Therefore, the minimum tumor-clearance parameter is $\mathcal{R}_{0}=r_{2} / r_{2}^{\star}$ and clearing the tumor requires the achievement of the inequality $\mathcal{R}_{0}<1$. It is equivalent to the following set of inequalities

$$
s_{1}>\frac{\mu_{1} \mu_{2}-\theta_{1} s_{2}}{\mu_{2} \alpha} r_{2}:=s_{1}^{\star}, \quad s_{2}<\frac{\mu_{1} \mu_{2}}{\theta_{1}}:=s_{2}^{\star} .
$$




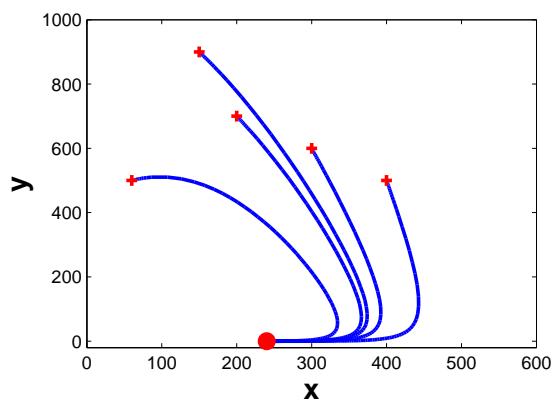

(a) Tumor-free equilibrium $\left(r_{2}=1\right.$ and $\alpha=$ $\left.5 * 10^{-3}\right)$

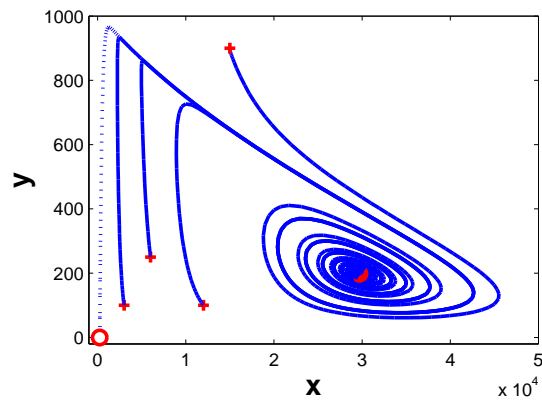

(c) One persistent-tumor equilibrium at little $\alpha\left(r_{2}=1\right.$ and $\left.\alpha=2.7 * 10^{-5}\right)$

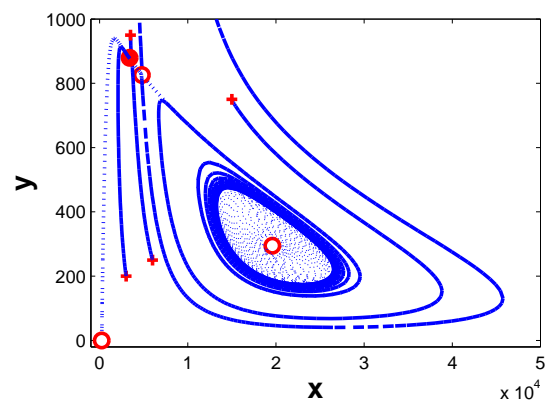

(e) Three persistent-tumor equilibria with a stable limit cycle $\left(r_{2}=0.5\right.$ and $\left.\alpha=1.8 * 10^{-5}\right)$

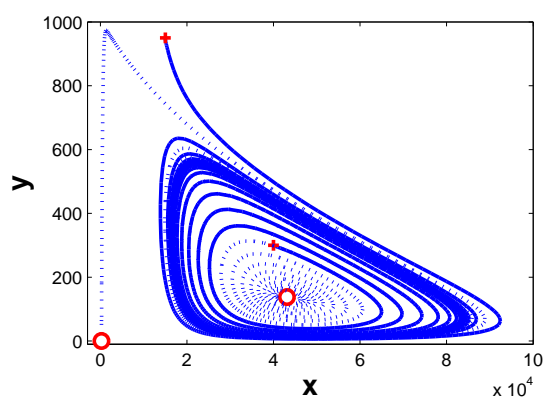

(b) One persistent-tumor equilibrium with a stable limit $\operatorname{cycle}\left(r_{2}=1\right.$ and $\left.\alpha=2 * 10^{-5}\right)$

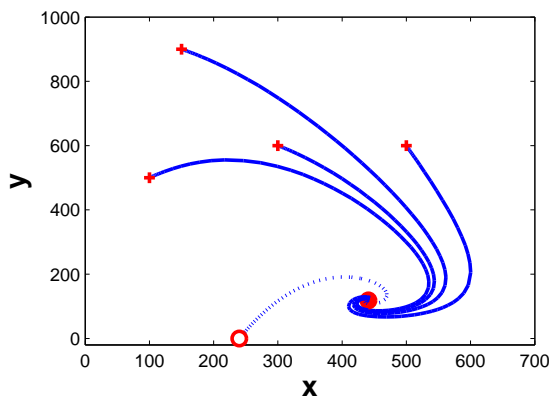

(d) One persistent-tumor equilibrium $\left(r_{2}=1\right.$ and $\alpha=2 * 10^{-3}$ )

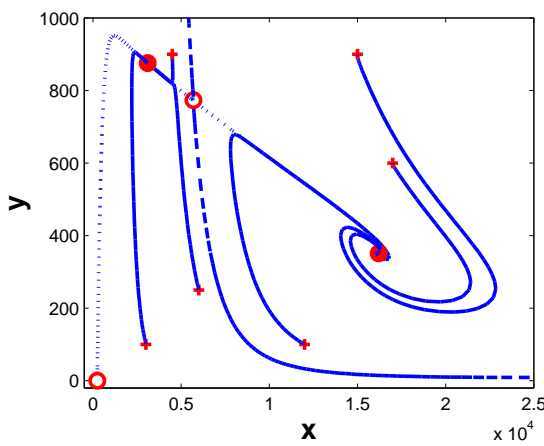

(f) Three persistent-tumor equilibria $\left(r_{2}=1\right.$ and $\alpha=4 * 10^{-5}$ )

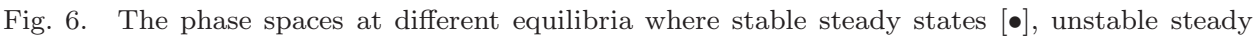
states $[\mathrm{O}]$, stable manifold [- - ], unstable manifold [. . .] and initial conditions [+]. (with immunotherapy with both ACI and IL-2, see Table 1 and $s_{1}=40, s_{2}=10$.) 


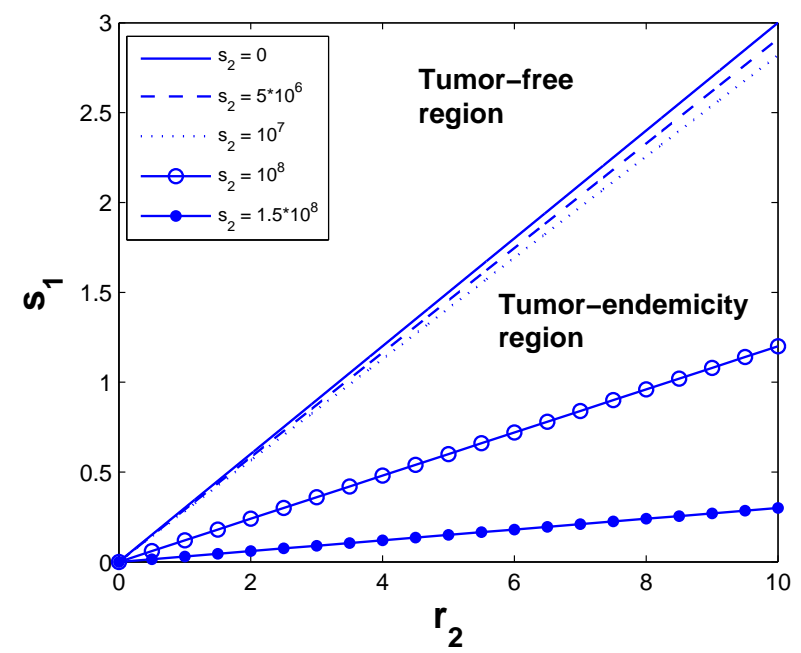

Fig. 7. The critical $s_{1}^{\star}$ as a function of $r_{2}$ for several levels of $s_{2}$. For values of $s_{1}$ above the threshold $s_{1}^{\star}$, the tumor cells do not exist (with $\alpha=0.5556$ ), see Table 1 .

Hence clearing the tumor depends mainly on the concentration of treatments: the external source of effector cells $s_{1}$ and the treatment $s_{2}$ which represents the external input of IL-2. If $s_{2}=0$, then the tumor can be cleared by treatment with adoptive cellular immunotherapy alone, $s_{1}>\left(\mu_{1} / \alpha\right) r_{2}$. However, for $s_{1}=0$, then the inequality $r_{2}<r_{2}^{\star}$ can not be held and therefore, it is impossible to treat cancer by IL-2 alone. However, a strategy based on using both adoptive immunotherapy and IL-2 with concentrations $s_{2}<s_{2}^{\star}$ and $s_{1}>s_{1}^{\star}$ could be used to clear the tumor; See Figure 7. We arrive to the following corollary.

Corollary 5.1. In the tumor-clearance problem we have the following three cases:

(1) if $s_{1}=0$, the tumor could never be cleared,

(2) if $s_{2}=0$, the tumor could be cleared by adding an external source of effector cells with concentration slightly above $s_{1}^{\star}=\frac{\mu_{1}}{\alpha} r_{2}$,

(3) if $s_{1} \neq 0$ and $s_{2} \neq 0$, then the tumor could be cleared with concentrations $s_{2}<\frac{\mu_{1} \mu_{2}}{\theta_{1}}$ and $s_{1}>\frac{\mu_{1} \mu_{2}-\theta_{1} s_{2}}{\mu_{2} \alpha} r_{2}$.

\section{Summary and Conclusion}

In this paper we introduced a family of models (ODEs and DDEs) to describe tumorimmune system dynamics. The qualitative and evolution of the models have been displayed with different values of the parameters $\alpha$ (the rate of tumor cells predated by the effector cells) and $r_{2}$ (the maximal growth rate of the tumor cells population). Although the underlying models are simple, they displays very rich dynamics and gives a good picture for the phenomena of real interaction of tumor growth and immunotherapy. The minimum tumor-clearance parameter $\mathcal{R}_{0}$ has been expressed 
in terms of the ratio between the tumor-growth rate and the critical tumor-growth rate. The cases at which the tumor can be cleared are summarized in the Corollary 5.1. The obtained results can help to gain a better understanding of interaction mechanisms, and make predictions; determine and evaluate control strategies and convey more general insight to biologists.

The obtained numerical results (have been obtained by semi-implicit RK methods $^{20}$ ) demonstrate that the system with time delay exhibits richer complex dynamics, such as quasiperiodic and chaotic patterns, compared with models without memory or after-effect. The sealy states of DDEs models are similar to the steady states of ODEs models. We shall extend this work to investigate the stability and qualitative and bifurcation analysis of more sophisticated models of delay differential equations with tumor cells-immunotherapy interactions.

\section{Acknowledgement}

This work was supported by UAE University (UAE) and Helwan University (EGYPT).

\section{References}

1. J. Adman and N. Bellomo, A survey of models on tumor immune systems dynamics, Birkhauser, Boston, 1996.

2. J. C. Arciero, D. E. Kirschner and T. L. Jackson, A mathematical model of tumorimmune evasion and siRNA treatment, Disc. Cont. Dyn. Syst. 4(1) (2004) 39-58.

3. L. Arlotti, N. Bellomo and E. De Angelis, Generalized kinetic (Boltzman) models: mathematical structures and application, Math. Models Method Appl. Sci. 12 (2002) $567-592$.

4. R. A. Beaumont and R. S. Pierce, The Algebraic Foundations of Mathematics. Reading, MA: Addison-Wesely, 1963.

5. N. Bellomo and L. Preziosi, Modeling and mathematical problems related to tumor immune system interactions, Math. comp. Modelling 31 (2000) 413-452.

6. N. Bellomo and M. Pulvirenti (Eds.), Modeling in Applied Sciences: A Kinetic Theory Approch, Birkhsher, 2000.

7. R. J. De Boer, P. Hogeweg, F. J. Dullens, R. A. De Weger and W. DenOtter, Macrophage $\mathrm{T}$ lymphocyte interactions in the antitumor immune response: A mathematical model, J. Immunol., 134(4), (1985) 2748-2758.

8. C. Delisi and A. Rescigno, Immune surveillance and neoplasia-I:A minimal mathematical model, Bull. Math. Biol., 39 (1977), 201-221.

9. L. G. De Pillis, W. Gu and A. E. Radunskaya, Mixed immunotherapy and chemotherapy of tumors:modelling, applications and biological interpretations, J. Theor. Biol. 238 (2006) 841-862.

10. V. T. DeVita, T. S. Lawrence, S. A. Rosenberg, R. A. DePinho and R. A. Weinberg, Cancer: Principles \& Practice of Oncology, 8th edition, Lippincott Williams and Wilkins, Philadelphia, 2008.

11. M. Galach, Dynamics of The Tumor-Immune System Comptition - The Effect of Time Delay, Int. J. Appl. Math. Comput. Sci., 13(3) (2003) 395-406.

12. C. Gallagher, M. Peckham, H. M. Pinedo and U. Veronesi, Oxford Textbook of Oncology, Oxford Medical Publications, Oxford, 1995. 
13. O. G. Isaeva and V. A. Osipov, Modelling of anti-tumor Immune response: Immunocorrective effect of weak centimeter electromagnetic waves, J. Comput. Math. Meth. Medicine, (2008) July 24,23.

14. B. Joshi, X. Wang, X. Banerjee, H. Tian, A. Matzavinos and M. A. J. Chaplain, On immunotherapies and cancer vaccination protocols: a mathematical modelling approach., J Theor. Biol., 259(4), (2009) 820-827.

15. D. Kirschner and J. C. Panetta, Modeling immunotherapy of the tumor immune interaction, J. Math. Biol. 37 (1998) 235-252.

16. V. A. Kuznetsov and M. A. Taylor, Nonlinear dynamics of immunogenic tumors: parameter estimation and global bifurcation analysis, Bull. Math. Biol. 56(2) (1994) $295-321$.

17. D. Liu, S. Ruan and D. Zhu, Bifurcation analysis in models of tumor and immune system interactions, DCDSB 12(1) (2009) 151-168

18. H. Ortega and A. Acosta, A model for tumor catastrophe in the presence of immunocompetent cells, CIENCIA 13(2) (2005) 134-146.

19. F. A. Rihan, Delay Differential Models in Dynamic Diseases, The Proceedings of the International Conference on Bioinformatics and Computational Biology 2010, Hawaii, USA, March 24-26 (2010), pp. 73-79.

20. F. A. Rihan, E. H. Doha, M. I. Hassan and N. M. Kamel, Numerical Treatments for Volterra Delay Integro-Differential Equations, Computational Methods in Applied Mathematics, 9(3) (2009) 292-308.

21. M. Safan, H. Heesterbeek and K. Dietz, The minimum effort required to eradicate infections in models with backward bifurcation, J. Math. Biol. 53, (2006) 703-718.

22. R. Yafia, Hopf bifurcation analysis and numerical simulations in an ODE model of the immune system with positive immune response, Nonlinear Analysis: Real World Applications, 8 (2007) 1359-1369.

23. R. Yafia, Hopf bifurcation in a delayed model for tumor-immune system competition, SIAM J. Appl Math., 67(6) (2007) 1693-1703. 